\title{
Coincident Salmonella Infections and Ulcerative Colitis: Problems of Recognition and Management
}

\author{
M. W. DRONFIELD, J. FLETCHER, M. J. S. LANGMAN
}

British Medical fournal, 1974, 1, 99-100

\section{Summary}

Five cases of coincident salmonellosis and ulcerative colitis are described. In three the diagnostic combination was recognized late. If corticosteroid therapy is given for the colitis once the combination is recognized, then simultaneous systemic antibiotic cover is advisable. One of the five patients died during corticosteroid therapy alone with salmonella septicaemia due to a usually noninvasive organism.

\section{Introduction}

Ulcerative colitis and salmonella infections of the bowel are both quite common disorders, and it is therefore not surprising that they should occasionally coincide in the same patient. This combination, however, has not received attention in the recent medical literature. We have seen five people with salmonella infections who also had typical clinical characteristics of ulcerative colitis. We report these because recognition of the combination was often slow, and satisfactory management was correspondingly delayed. One patient ultimately died with extensive colitis and a potentially preventable salmonella septicaemia.

\section{Case Reports}

Case 1.-A 55-year-old man presented in August 1970 with a three-week history of bloody diarrhoea since returning from holiday in Spain. Salmonella typhimurium was isolated from the first three stool cultures taken, and he was treated for infective diarrhoea with oral kanamycin. Stool cultures became persistently negative but his symptoms persisted. Treatment was changed to co-trimoxazole, without benefit. After three weeks in hospital he was obviously deteriorating, the haemoglobin having fallen from normal to 6.0 $\mathrm{g} / 100 \mathrm{ml}$ due to increasing rectal blood loss. Sigmoidoscopy at this time showed severe proctitis with a friable and bleeding mucosa, and on rectal biopsy a non-specific inflammatory cell infiltrate was found while barium enema showed gross abnormality throughout the colon, with submucosal ulceration and extensive pseudopolyposis. The diagnosis of ulcerative colitis was made, and treatment with oral prednisolone $\mathbf{4 0} \mathrm{mg}$ daily started. This was now four weeks after his last positive stool culture, and there had been 15 negative cultures since. Infection was therefore assumed to be no longer a problem. Response to treatment was wholly satisfactory at first and he became virtually asymptomatic after a few days. However, 12 days after starting steroids his symptoms returned, with bloody diarrhoea, vomiting, abdominal pain, and pyrexia. Salm. typhimurium was once again isolated from the stools and also from three consecutive blood cultures. Treatment was started with parenteral ampicillin and later parenteral chloramphenicol. He continued to deteriorate, developing a silent, distended, tender abdomen, and he died 10 days after developing salmonella septicaemia. Necropsy showed a generalized peritonitis. The colon was dilated and the

City Hospital, Nottingham, NG5 1PB

M. W. DRONFIELD, M.B., M.R.C.P., Medical Registrar

M. FLETCHER, M.D., M.R.C.P., Consultant Physician

M. J. S. LANGMAN, M.D., F.R.C.P., Professor of Therapeutics mucosa showed acute ulceration wth pseudopolyps, the appearances being those of ulcerative colitis with perforation, though the site of perforation was not identified. Histology of the colon was compatible with ulcerative colitis. Salm. typhimumium was grown from the gall bladder and spleen.

Case 2.-An 18-year-old man presented in August 1971 with a history of two weeks' severe bloody diarrhoea, abdominal pain, and occasional vomiting. There was no past history of bowel disturbance or other significant illness. On examination he was pale and dehydrated, and there was pronounced lower abdominal tenderness. Haemoglobin was $9.0 \mathrm{~g} / 100 \mathrm{ml}$ and W.B.C. $15,000 / \mathrm{mm}^{3}$. Salm. typhimurium was grown from several stool cultures. He was treated for salmonellosis with oral kanamycin and parenteral fluids. Stool cultures became negative but his symptoms worsened. Two weeks after admission the haemoglobin had fallen to $6.0 \mathrm{~g} / 100 \mathrm{ml}$. An underlying colitis was suspected and further investigation undertaken. Sigmoidoscopy showed a severe proctitis and on rectal biopsy an inflammatory cell infiltrate was found. Barium enema showed an abnormal mucosal pattern of the entire colon with numerous small irregularities, the appearances being those of acute ulcerative colitis. Three weeks after admission treatment was started for ulcerative colitis, consisting of oral prednisolone, sulphasalazine, and blood transfusion. In addition, parenteral ampicillin was given because of the risk of salmonella septicaemia. He improved rapidly, and on discharge three weeks later he was virtually asymptomatic. Shortly after starting steroids his stool cultures again grew Salm. typhimurium, but blood cultures were negative and his progress was uninterrupted. He has had no symptoms in the two years since this illness. Repeat barium enema 15 months after his illness showed an entirely normal colon.

Case 3.-A 34-year-old man presented in August 1971 with a three-week history of profuse watery diarrhoea not containing blood. There were also quite severe abdominal pain, feverishness, and infrequent vomiting. He gave no past history of bowel disturbance. On examination he was pyrexial but no other abnormality was found. Haemoglobin was $14.0 \mathrm{~g} / 100 \mathrm{ml}$ and W.B.C. $15,000 \mathrm{~mm}^{3}$. Salm. agona was isolated from his stools. A diagnosis of salmonellosis

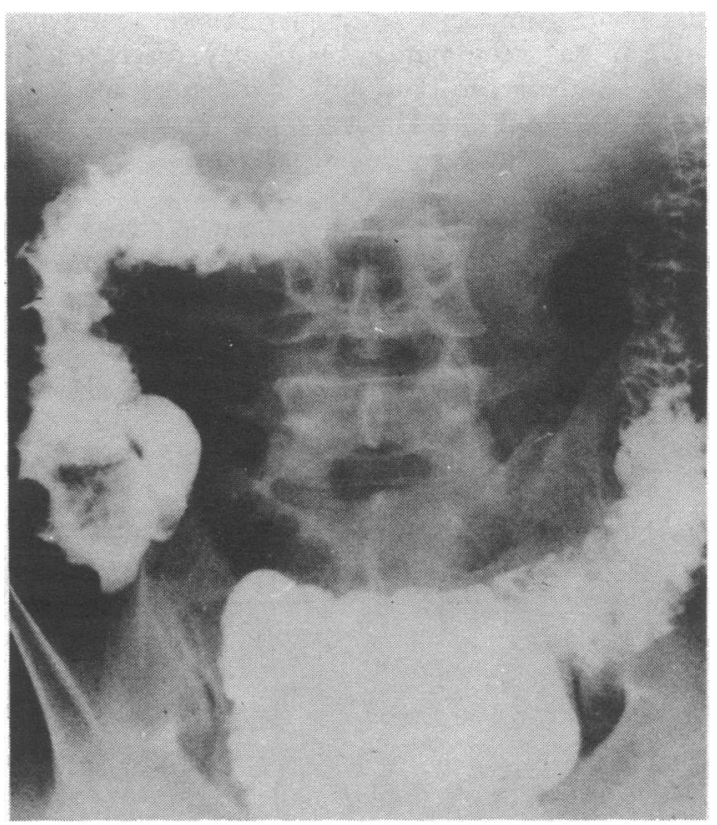

$X$-ray appearances of severe ulcerative colitis in case 3. 
was therefore made, and treatment started with oral kanamycin Stool cultures became negative but he did not improve, having continuing diarrhoea which was now noted to contain much blood. Haemoglobin had fallen to $9.0 \mathrm{~g} / 100 \mathrm{ml}$ three weeks after admission. An underlying colitis was suspected and sigmoidoscopy showed a severe proctitis and on rectal biopsy an inflammatory cell infiltrate of the lamina propria was found. Barium enema showed a grossly abnormal appearance with pseudopolyposis and submucosal ulceration, the appearances being those of severe ulcerative colitis (see fig). Three weeks after the last positive stool culture treatment was started for ulcerative colitis with oral prednisolone. Parenteral antibiotics were not given. He made an excellent recovery over the next few weeks and has been asymptomatic for nearly two years. However, a barium enema 18 months later still showed pseudopolyposis and diminution of haustral pattern.

Case 4.-A 45-year-old woman had frequent episodes of mild bloody diarrhoea between 1950 and 1969 but never sought medical advice. In 1969, however, she had a severe attack of bloody diarrhoea and was found at that time to have severe proctitis on sigmoidoscopy with a normal barium enema. Stool cultures were negative. In November 1972 she presented with her worst ever symptomatic attack, consisting of profuse bloody diarrhoea and lower abdominal discomfort over the past two months. Sigmoidoscopy showed severe proctitis and a rectal biopsy showed an inflammatory cell infiltrate. Barium enema revealed ulceration and narrowing of the pelvic colon and rectum, strongly suggesting ulcerative colitis. An unnamed salmonella (antigenic structure $0: 4,12 . \mathrm{H}: \mathrm{d}$ monophasic) was grown from her stools. Treatment was with rectal and systemic steroids and parenteral ampicillin. She improved rapidly over the next two weeks and stool cultures becames persistently negative. She has been well since.

Case 5.-A 20-year-old man was admitted to hospital in February 1973. He complained of mild diarrhoea for three months which had become severe and bloody over the past two weeks and was accompanied by abdominal pain. There was no past history of bowel disturbance. On examination he was pale and pyrexial, and there was slight abdominal tenderness. Haemoglobin was $9.0 \mathrm{~g} / 100$ $\mathrm{ml}$. Sigmoidoscopy showed a severe proctitis and treatment was started for ulcerative colitis with oral prednisolone. Two days later the stool culture report became available, showing a growth of Salm. brednei. Parenteral ampicillin was therefore added. Barium enema was attempted. He did not retain the barium but postevacuation films showed an abnormal pattern in the descending and sigmoid colon. On the combined therapy he made a complete recovery and has been asymptomatic since.

\section{Discussion}

It may be argued that the illness in some of these patients was due entirely to salmonella infection. Severe acute dysenteric infection can be accompanied by bloody diarrhoea, and the lower bowel mucosa can both appear inflamed and contain an inflammatory cell infiltrate. However, the illnesses of our patients all had features which are difficult to reconcile with simple, even if severe, salmonella dysentery.

In case 1 (the only patient to die) the whole large bowel had typical features of ulcerative colitis with severe inflammation, pseudopolyp formation, ragged linear ulceration, and extensive mucosal stripping from the submucosa. In case 3 anaemia was severe, and barium enema examination during the attack and eighteen months later showed the typical pattern observed in chronic ulcerative colitis. Despite reversion to negative stool cultures bloody diarrhoea worsened in case 2, the haemoglobin continued to fall, and a response was obtained only when he was treated for ulcerative colitis. A previous history typical of ulcerative colitis was obtained in case 4 , and barium enema showed the characteristic changes of chronic proctosigmoid disease. Finally, in case 5, the combination of ulcerative colitis and salmonellosis was suggested by the profuse bloody diarrhoea for two weeks before admission, a pronounced anaemia, severe inflammatory change seen at sigmoidoscopy, and an abnormal result on barium enema.

There is little doubt that salmonella septicaemia was the major factor causing the death of our first patient. At the time it was thought that multiple negative stool cultures for Salm. typhimurium, not usually an invasive organism, made treatment with corticosteroid drugs alone logical. Oral antibiotic treatment is ineffective in eliminating salmonellae from the bowel, and may prolong their excretion and precipitate free transfer of resistance factors between organisms (Dixon, 1965; Association for Study of Infectious Diseases, 1970). Parenteral treatment with appropriate antibiotics is, however, usually effective in treating systemic salmonellosis (Sleet et al., 1964; Robertson et al., 1968), but probably failed in our first case because of high dosage corticosteroid therapy in a severely ill colitic patient. Three of the remaining four patients were treated concurrently with systemic antibiotics and corticosteroids as soon as the presence of ulcerative colitis was recognized, and recovered uneventfully. Though the fourth patient (case 3) recovered with steroid treatment alone, experience with our first patient leads us to suggest that systemic antibiotic cover with ampicillin is a wise precaution in patients receiving corticosteroid drugs for ulcerative colitis recognized in the presence of, or shortly after, infection with salmonellae, even with usually non-invasive organisms.

Stool culture is not always undertaken in patients with acute attacks of ulcerative colitis, nor is this latter diagnosis usually considered until late in patients with salmonella diarrhoea even though bloody diarrhoea is prominent. It may be relevant that the first three patients, in whom the diagnosis of ulcerative colitis was delayed, were all admitted initially to an infectious diseases hospital where the expectation of infectious disease is high and of underlying chronic medical disease low. Our experience again leads us to suggest that the diagnosis of ulcerative colitis should be considered promptly if apparent salmonella diarrhoea is persistently and profusely blood stained; or if a patient with ulcerative colitis has a sudden onset of profuse diarrhoea the stools should be cultured.

\section{References}

Association for the Study of Infectious Diseases (1970). Lancet, 2, 1159. Dixon, J. M. S. (1965). British Medical fournal, 2, 1343. Robertson, R. D W Wahab, M. F. A., and Raasch, F. O., (1968). New England fournal of Medicine, 278,171 .

Sleet, R. A., Sangster, G., and Murdoch, J. McC. (1964). British Medical fournal, 1,148 . 